\title{
Microwave transmission through metallic hole arrays: Surface electric field measurements
}

\author{
Bo Hou, Zhi Hong Hang, Weijia Wen, ${ }^{\text {a) }}$ C. T. Chan, and Ping Sheng \\ Department of Physics, The Hong Kong University of Science and Technology, Clear Water Bay, Kowloon, \\ Hong Kong
}

(Received 25 July 2006; accepted 17 August 2006; published online 27 September 2006)

\begin{abstract}
The authors investigate the enhanced microwave transmission through a metal plate perforated by a square lattice of subwavelength holes, predicted to occur as a structure factor resonance phenomenon [F. J. Gracía de Abajo and J. J. Sáenz, Phys. Rev. Lett. 95, 233901 (2005)]. By probing the surface electric field on the metallic plate at the peak transmission frequency, they establish the similarities and differences between the structure factor resonance and surface plasmon. (C) 2006 American Institute of Physics. [DOI: 10.1063/1.2357942]
\end{abstract}

In the past few years, phenomena of enhanced transmissions of electromagnetic (EM) waves through subwavelength apertures have received intensive studies. ${ }^{1-8}$ The physical mechanism responsible for the extraordinary transmissions was initially believed to be resonant excitations of surface plasmon (SP) on the holed metal films, ${ }^{9-14}$ though the true role of SP has been questioned subsequently. ${ }^{15,16}$ Recently, transmission enhancement phenomena in terahertz and millimeter-wave frequency regimes have been experimentally demonstrated. ${ }^{17-19}$ In these frequency domains, metals serve ideally as perfect conductors and therefore would not be able to support SP on their surfaces. Pendry and coworkers proposed that even perfect conductors, once textured with periodic structures, can support a type of SP-like modes to resolve the debate over extraordinary transmissions through subwavelength holes. ${ }^{20,21}$ Corresponding experimental investigations were implemented in microwave frequencies by Hibbins et al. that verified the theoretical prediction. ${ }^{22}$ A combination of subwavelength holes and SPlike modes on perfectly conducting surfaces thus opens new interest to realize extraordinary transmissions of microwaves through structured conductors. More recently, Gracía de Abajo et al. $^{23}$ and Gracía de Abajo and Sáenz ${ }^{24}$ have shown that the enhanced transmission phenomenon in perfect conductor hole arrays has its origin in the resonance-induced field enhancement arising from the periodic structure factor, which fundamentally differs from the surface plasmon, as the latter involves the inertial mass of electrons and should display nonzero surface tangential electric field.

In this letter, we study the microwave transmission through a metal plate perforated by a square lattice of small holes at the frequencies of $10-18 \mathrm{GHz}$, with the intention of examining the similarities and differences between the structure factor resonance and the surface plasmon. In particular, we measured the surface electric fields on the transmission side of the metallic plate, at the peak transmission frequency, so as to obtain a more detailed description of the transmission phenomenon. For a reasonably thick metallic plate, a transmission peak significantly greater than that due to the hole filling ratio background was observed at the frequency slightly lower than Wood's anomaly frequency. We find that

\footnotetext{
${ }^{a}$ Author to whom correspondence should be addressed; electronic mail: phwen@ust.hk
}

the surface tangential electric field is nonzero only in the hole region, as expected. But the positive and negative charge densities as measured by the normal electric field can extend to regions intermediate between the holes, making it similar to a surface plasmon. Experimental results are shown to agree well with finite-difference time-domain (FDTD) simulations.

Consider a beam of microwaves impinging normally on a square lattice of small holes, as shown in Fig. 1(a). The sample is a $0.5 \mathrm{~mm}$ thick brass plate with a square array of $15 \times 15$ holes, each being $8.0 \mathrm{~mm}$ in diameter with a lattice constant $a=20 \mathrm{~mm}$. The size parameters were chosen in order to obtain a strong transmitted signal while preventing waveguiding transmission through the holes in the measurement frequencies. It is noted that an $8.0 \mathrm{~mm}$ hole has the cutoff frequency of $\sim 22 \mathrm{GHz}{ }^{25}$ Microwave transmission
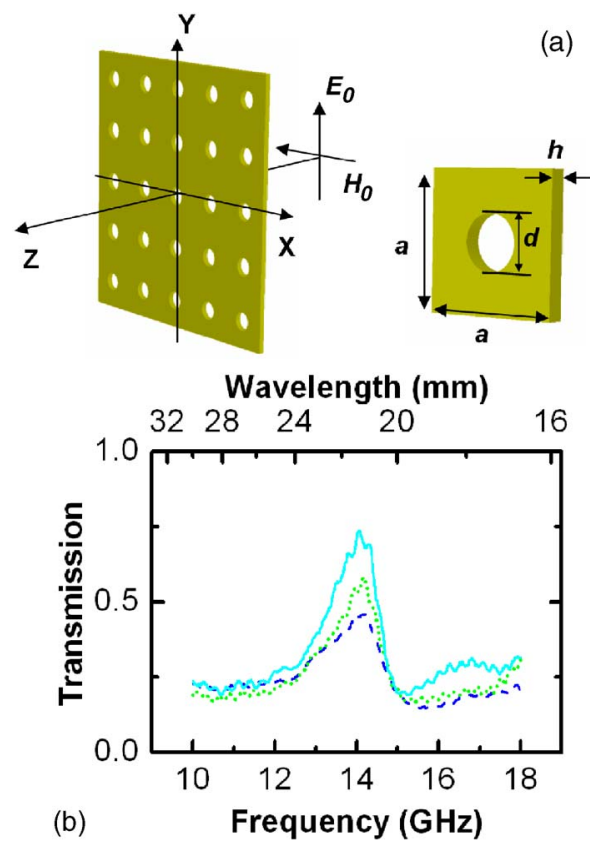

FIG. 1. (Color online) (a) Schematics of the sample and its unit cell, where $a$ is the lattice constant, $d$ the diameter of the hole, and $h$ the thickness of the plate. Microwaves are normally incident along the $z$ axis with $E_{0}$ along the $y$ axis and $H_{0}$ along the $x$ axis. (b) Transmission spectra for three samples. The three samples have the same lattice of holes, $a=20 \mathrm{~mm}$ and $d$ $=8.0 \mathrm{~mm}$, but different thicknesses, $h=0.5 \mathrm{~mm}$ (solid line), $1.0 \mathrm{~mm}$ (dot line), and $2.0 \mathrm{~mm}$ (dash line). 

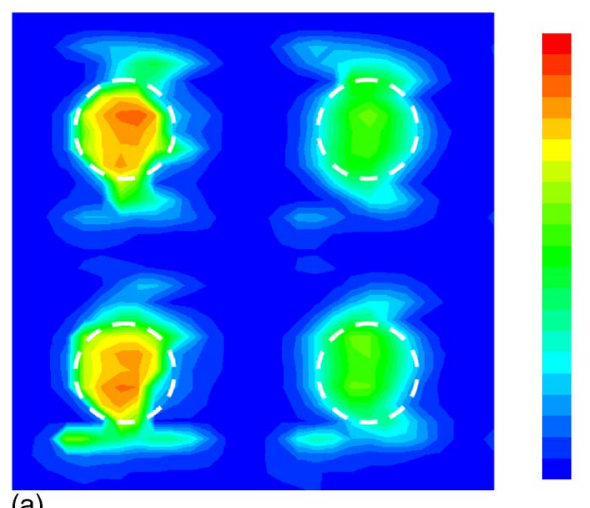

(a)

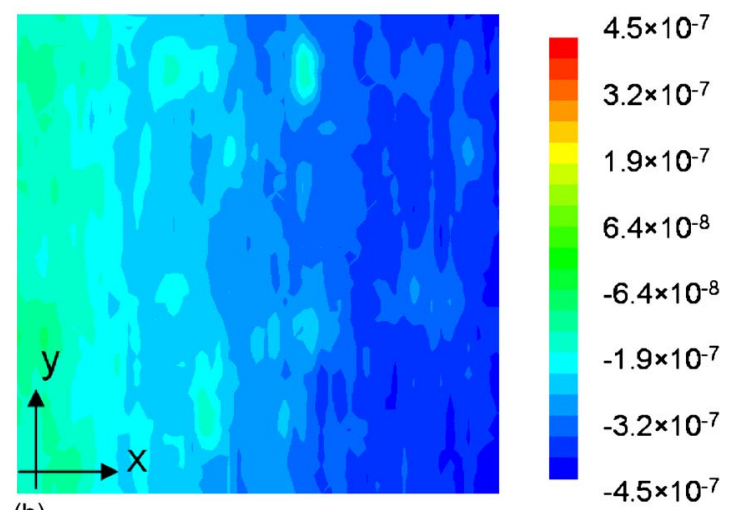

(b)

$-4.5 \times 10^{-7}$

FIG. 2. (Color online) Scanning maps of (a) $\left|E_{y}\right|$ and (b) $\left|E_{x}\right|$ on the exit surface of the sample at the peak frequency of $14.10 \mathrm{GHz}$. The scanning region measures $40 \times 40 \mathrm{~mm}^{2}$ and includes $2 \times 2$ holes as outlined. The incident electric field is polarized along the $y$ direction and the magnetic field along the $x$ direction. Negative values shown here are due to the signal intensity detected that is lower than the zero of the instrument.

spectra were recorded by a network analyzer (Agilent $8720 \mathrm{ES}$ ). Two identical horn antennas were used to generate and receive EM waves, separated by a distance of $\sim 140 \mathrm{~cm}$. The sample was placed between two horns at a distance $\sim 40 \mathrm{~cm}$ from the receiving horn. The measured spectra have been normalized to the transmission value without the sample.

Figure 1(b) shows the experimental measurement, where a transmission peak $(\sim 75 \%)$ at $14.10 \mathrm{GHz}$ and a dip $(\sim 20 \%)$ at $15.07 \mathrm{GHz}$ are seen. The dip corresponds to Wood's anomaly which should occur at the wavelength $\lambda$ $=a$. The peak is located at the wavelength of $21 \mathrm{~mm}$, and this value is larger than the hole itself as well as the cutoff wavelength. For such long wavelength, the wave becomes evanescent when traveling inside the holes and the resultant transmission would be expected as low as the prediction of Bethe's theory. ${ }^{26}$ However, the peak transmission is huge compared to such value and is even larger than the geometrical filling ratio of the holes (i.e., assuming 100\% transmission through the hole area), which means that not only the EM energy impinging directly on the holes gets transmitted but also part of the energy shining the metallic surface is converted into transmission. In the experiment, we also tested different samples with thicknesses of 1.0 and $2.0 \mathrm{~mm}$ [see Fig. 1(b)]. For even thicker samples, the peak transmission value is seen to decay, but the frequency position remains unchanged.

The distribution of the near field on the surface of the sample was probed at the peak transmission frequency. We Downloaded 28 Jun 2007 to 143.89.104.110. Redistribution subject


FIG. 3. (Color online) (a) Scanning map of $\left|E_{z}\right|$ on the exit surface of the sample at the peak frequency of $14.10 \mathrm{GHz}$. The scanning region measures $40 \times 40 \mathrm{~mm}^{2}$ and includes $2 \times 2$ holes centered at the coordinates $(10,10)$, $(30,10),(10,30)$, and $(30,30)$, respectively. The incident electric field is polarized along the $y$ direction and the magnetic field along the $x$ direction. (b) The variations of the relative phase of $E_{z}$ component along two axes, as denoted in (a). (c) A color plot combining both the magnitude and phase of the measured charge density as deduced from $E_{z}$ from the previous two figures, (a) and (b). (d) FDTD simulation results to be compared with (c).

replaced the receiving horn by a detector behind the sample. The detector was a waveguide/coax adaptor (WR62, 12.4-18 GHz), mounted on a moving stage and allowed to do a point-by-point scanning within the $x-y$ plane, with a spatial resolution of $1 \times 1 \mathrm{~mm}^{2}$. The transmitting horn was connected with a signal generator (HP83711B) to stimulate a single frequency wave, while the detector was connected to a power meter to sense the field intensity. The detector, with polarization initially set along the $y$ direction, was brought to a distance of $0.2 \mathrm{~mm}$ from the surface on the transmission side of the sample. The scanned region is a $40 \times 40 \mathrm{~mm}^{2}$ square with $2 \times 2$ holes. The scanning map of the tangential electric field is shown in Fig. 2(a) for the $E_{y}$ intensity on the exit surface of the sample. The $E_{y}$ component is seen to be concentrated almost entirely on the holes, with negligibly small values on the metallic part of the surface. By adjusting the detector's polarization to be along the $x$ direction, the scanned distribution of $E_{x}$ is shown in Fig. 2(b). It is seen that there is no $E_{x}$ component across the whole region, except for a variation of the intensity profile attributable to the nonuniformity of the incident wave front.

To probe the $E_{z}$ distribution, we used a $16 \mathrm{~mm}$ monopole antenna as the detector and orientated the monopole normal to the exit surface. The resulting intensity map of $E_{z}$ is shown in Fig. 3(a) and revealed that four bands of high intensity are formed along the $x$ direction and that these bands are separated by some blue strips along the $y$ direction. According to the boundary condition $\mathbf{n} \cdot \mathbf{E}=\sigma / \varepsilon_{0}$, where $\sigma$ is the surface charge density, ${ }^{25}$ the high intensity regions are associated with surface charges, with the blue stripes implying zero surface charge. Therefore, we are able to determine the magnitude of surface charges through detecting $\left|E_{z}\right|$. In addition, we measured the phase of $E_{z}$ along two selected dashed lines as shown in Fig. 3(a) because the phase would indicate whether these surface charges are positive or negative. The results are shown in Fig. 3(b). Along line 1, very tiny phase change was observed. In contrast, a phase change, close to $\pi$ jump, was seen every $10 \mathrm{~mm}$ along line 2 . So we infer from to AIP license or copyright, see http://apl.aip.org/apl/copyright.jsp 
this result that each band in Fig. 3(a) is composed of charges of the same sign and the charges in two neighboring bands are opposite. From Figs. 3(a) and 3(b), we obtained a periodic distribution of surface charges and such distribution is consistent with the dipolar nature of subwavelength holes revealed from the tangential electric field components in Fig. 2. Seemingly, each hole behaves like a dipole and these "dipoles" are flanged by opposite surface charges outside the holes and these periodic charges establish a standing wave resonance on the surface when the incident wavelength is close to the lattice constant. This picture is very similar to that of the surface plasmon. However, where it differs from the surface plasmon picture is the region between the holes, where there is no tangential electric field. Here the charge density is seen to diminish from the high values on two sides of the holes, along the $y$ direction. Ultimately, the surface charges radiate the EM energy towards the far field with the characteristic wavelength $\lambda \approx a$. As discussed in Refs. 23 and 24, a dipole (hole) array will fully reflect (transmit) EM waves because of inducing a coherent structural resonance at wavelength a bit larger than the periodicity of arrays, implying enhanced transmission for the complementary structure, i.e., the metallic hole array. Therefore the microwave transmission enhancement here is not related to SP but to a surface charge resonance determined by periodicity, although there is definitely a resemblance in the surface charge density distribution.

Combining the magnitude and phase information, we plot them together in Fig. 3(c), which lends itself to direct comparison with FDTD simulations in Fig. $3(\mathrm{~d}) .{ }^{27}$ It is seen that the agreement is satisfactory.

The authors are thankful to Mei Jun and Qiu Cunyin for beneficial discussions. This project is supported by Hong Kong RGC Grant Nos. CA02/03.SC01 and HKUST603603.
${ }^{1}$ T. W. Ebbesen, H. J. Lezec, H. F. Ghaemi, T. Thio, and P. A. Wolff, Nature (London) 391, 667 (1998).

${ }^{2}$ Y. Takakura, Phys. Rev. Lett. 86, 5601 (2001).

${ }^{3}$ F. Yang and J. R. Sambles, Phys. Rev. Lett. 89, 063901 (2002).

${ }^{4}$ H. E. Went, A. P. Hibbins, J. R. Sambles, C. R. Lawrence, and A. P. Crick, Appl. Phys. Lett. 77, 2789 (2000).

${ }^{5}$ F. I. Baida, D. V. Labeke, G. Granet, A. Moreau, and A. Belkhir, Appl. Phys. B: Lasers Opt. 79, 1 (2004).

${ }^{6}$ W. Fan, S. Zhang, B. Minhas, K. J. Malloy, and S. R. J. Brueck, Phys. Rev. Lett. 94, 033902 (2005).

${ }^{7}$ F. J. García-Vidal, E. Moreno, J. A. Porto, and L. Martín-Moreno, Phys. Rev. Lett. 95, 103901 (2005)

${ }^{8}$ L. Zhou, W. Wen, C. T. Chan, and P. Sheng, Phys. Rev. Lett. 94, 243905 (2005).

${ }^{9}$ H. F. Ghaemi, T. Thio, D. E. Grupp, T. W. Ebbesen, and H. J. Lezec, Phys. Rev. B 58, 6779 (1998).

${ }^{10}$ J. A. Porto, F. J. García-Vidal, and J. B. Pendry, Phys. Rev. Lett. 83, 2845 (1999).

${ }^{11}$ L. Martín-Moreno, F. J. García-Vidal, H. J. Lezec, K. M. Pellerin, T. Thio, J. B. Pendry, and T. W. Ebbesen, Phys. Rev. Lett. 86, 1114 (2001).

${ }^{12}$ W. L. Barnes, W. A. Murray, J. Dintinger, E. Devaux, and T. W. Ebbesen, Phys. Rev. Lett. 92, 107401 (2004).

${ }^{13}$ L. Martín-Moreno and F. J. García-Vidal, Opt. Express 12, 3619 (2004).

${ }^{14}$ W. L. Barnes, A. Dereux, and T. W. Ebbesen, Nature (London) 424, 824 (2003).

${ }^{15}$ Q. Cao and P. Lalanne, Phys. Rev. Lett. 88, 057403 (2002).

${ }^{16}$ H. J. Lezec and T. Thio, Opt. Express 12, 3629 (2004).

${ }^{17}$ H. Cao and A. Nahata, Opt. Express 12, 1004 (2004).

${ }^{18}$ F. Miyamaru and M. Hangyo, Phys. Rev. B 71, 165408 (2005).

${ }^{19}$ M. Beruete, M. Sorolla, I. Campillo, J. S. Dolado, L. Martín-Moreno, J. Bravo-Abad, and F. J. García-Vidal, Opt. Lett. 29, 2500 (2004).

${ }^{20}$ J. B. Pendry, L. Martín-Moreno, and F. J. García-Vidal, Science 305, 847 (2004).

${ }^{21}$ F. J. García-Vidal, L. Martín-Moreno, and J. B. Pendry, J. Opt. A, Pure Appl. Opt. 7, S97 (2005)

${ }^{22}$ A. P. Hibbins, B. R. Evans, and J. R. Sambles, Science 308, 670 (2005).

${ }^{23}$ F. J. García de Abajo, R. Gómez-Medina, and J. J. Sáenz, Phys. Rev. E 72, 016608 (2005).

${ }^{24}$ F. J. García de Abajo and J. J. Sáenz, Phys. Rev. Lett. 95, 233901 (2005).

${ }^{25}$ D. H. Staelin, A. W. Morgenthaler, and J. A. Kong, Electromagnetic Waves (Prentice Hall, Englewood Cliffs, NJ, 1994).

${ }^{26}$ H. A. Bethe, Phys. Rev. 66, 163 (1944).

${ }^{27}$ Simulations were performed using the software CONCERTO 3.5, Vector Fields Limited, England, 2003. 